\title{
On M-Asymmetric Semi-Open Sets and Semicontinuous Multifunctions in Bitopological Spaces
}

\author{
Levy K. Matindih* (1), Edwin Moyo \\ Department of Science \& Mathematics, School of Science, Engineering and Technology, Mulungushi University, Kabwe, Zambia \\ Email: *kahyamatindih99@gmail.com
}

How to cite this paper: Matindih, L.K. and Moyo, E. (2021) On M-Asymmetric SemiOpen Sets and Semicontinuous Multifunctions in Bitopological Spaces. Advances in Pure Mathematics, 11, 218-236. https://doi.org/10.4236/apm.2021.114016

Received: January 10, 2021

Accepted: April 5, 2021

Published: April 8, 2021

Copyright $\odot 2021$ by author(s) and Scientific Research Publishing Inc. This work is licensed under the Creative Commons Attribution International License (CC BY 4.0).

http://creativecommons.org/licenses/by/4.0/

\begin{abstract}
The purpose of this paper is to introduce the notions of $m$-asymmetric semiopen sets and $M$-asymmetric semicontinuous multifunctions defined between asymmetric sets satisfying certain minimal conditions in the framework of bitopological spaces. Some new characterizations of $m$-asymmetric semiopen sets and $M$-asymmetric semicontinuous multifunctions will be investigated and several fundamental properties will be obtained.
\end{abstract}

\section{Keywords}

$m$-Spaces, $m$-Semiasymmetric Open and Closed Sets, Upper and Lower $M$-Asymmetric Semicontinuous Multifunction

\section{Introduction}

The concept of topology is an important tool that has received considerable attention from many scholars in many fields of applied sciences and other branches of pure mathematics. Continuity and multifunctions on the other hand, which are basic notions in the theory of classical point set topology also play a vital role not only in the field of pure mathematics but also in applied mathematics. In this regard, several scholars have generalizations the notion of continuity in topological spaces using the weaker forms of open and closed sets the semiopen and semiclosed sets [1] [2] [3].

The concept of semiopen sets and semi-continuity of function first appeared in a paper by Levine [1] in the realm of topological spaces and this idea was then generalized and extended to the frameworks of bitopological spaces by Maheshwari and Prasad [2], and also by Bose [4]. In 1963, Berge [5] introduced the concepts of upper and lower continuous multifunctions and lately, Whyburn [6] 
also studied the general continuity of multifunctions in the realm of topological spaces. Smithson [7] and Popa [8] respectively then generalized these notions of multifunction continuity to the setting of bitopological spaces. In 2000, Popa and Noiri [9], introduced and studied the concepts of $m$-structures and $M$-continuous function as a function defined between topologies satisfying certain minimal conditions. They showed that the $M$-continuous functions have properties similar to those of continuous functions between topological spaces. With these ideas of multifunction continuity and $M$-continuous functions, Noiri and Popa [10] then introduced and studied upper and lower $M$-continuous multifunctions and showed how these functions have properties similar to those of upper and lower continuous functions and continuous multifunctions between topological spaces.

In this present paper, we introduce and investigate some basic characterizations and properties of $m$-asymmetric semiopen sets, $m$-asymmetric semiclosed sets and, upper and lower $M$-asymmetric semicontinuous multifunctions in the realm of bitopological spaces satisfying a minimal structure, which is a generalization of results of Noiri and Popa [10] and also Berge [5].

This paper is organized as follows. Section 2 presents some necessary preliminaries concerning semiopen sets, continuity and multifunctions on sets satisfying certain minimal structures. In Section 3, we investigate and study the concept of $m$-asymmetric semiopen and semiclosed sets in the realm of bitopological spaces, a generalition of semiopen and semiclose closed sets in [1] [2] and [4]. Section 4 presents and discusses some results of upper and lower $M$-asymmetric semicontinuous multifunctions as a generalized idea of upper and lower upper $M$-continuous multifunctions first introduced in [8] and then [10]. Section 5 gives some concluding remarks.

\section{Preliminaries and Basic Properties}

Throughout the section, we recall some basic notations and properties, which we refer the reader to ([1] [2] [3] [4] [7] [8] [9] [10] [11]).

By a bitopological space $\left(X, \mathscr{T}_{1}, \mathscr{T}_{2}\right)$ we mean a nonempty set $X$ on which are defined two topologies $\mathscr{T}_{1}$ and $\mathscr{T}_{2}$, this idea was first introduced by Kelly ([12]). In the sequel, $\left(X, \mathscr{T}_{1}, \mathscr{T}_{2}\right)$ or in short hand $X$ will mean a bitopological space unless stated. Given a bitopological space $\left(X, \mathscr{T}_{i}, \mathscr{T}_{j}\right), i, j=1,2$; $i \neq j$, the interior and closure of $A \subseteq X$ with respect to the topology $\mathscr{T}_{i}=\mathscr{T}_{j}$ is denoted by $\operatorname{Int}_{\widetilde{T_{i}}}(B)$ and $\mathrm{Cl}_{\widetilde{T}}(A)$ respectively.

Definition 2.1. Let $\left(X, \mathscr{T}_{i}, \mathscr{T}_{j}\right), i, j=1,2 ; i \neq j$ be a bitopological space, $A \subseteq X$.

(i) A is said to be $\cdot \mathscr{T}_{i} \cdot \widetilde{T}_{j}$-open if $A \in \mathscr{T}_{i} \cup \cdot \mathscr{T}_{j}$. The complement of an $\mathscr{T}_{i} \cdot \mathscr{T}_{j}-$ open set is a $\mathscr{T}_{i} \mathscr{T}_{j}$-closed set [12].

(ii) The $\mathscr{T}_{i} \cdot \mathscr{T}_{j}$-interior of $A$ denoted by $\operatorname{Int}_{\overparen{T_{i}}}\left(\operatorname{Int}_{\overparen{J_{j}}}(A)\right)$ (or $\left.\mathscr{T}_{i} \cdot \mathscr{T}_{j}-\operatorname{Int}(A)\right)$ is the union of all $\mathscr{T}_{i} \mathscr{T}_{j}$-open subsets of $X$ contained in $A$. Clearly, $A$ is $\mathscr{T}_{i} \cdot \mathscr{T}_{j}$ open provided $A=\operatorname{Int}_{\overparen{T_{i}}}\left(\operatorname{Int}_{\overparen{J_{j}}}(A)\right)$.

(iii) The $\cdot \mathscr{T}_{i} \cdot{ }_{j}$-closure of $A$ denoted by $C l_{\overparen{T}}\left(C l_{\overparen{J_{j}}}(B)\right)$ defined to be the 
intersection of all $\mathscr{T}_{i} \cdot T_{j}$-closed subsets of $X$ containing $A$. And

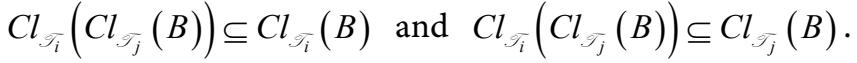

(iv) $A$ is said to be pairwise open if it is both $\mathscr{T}_{1} \mathscr{T}_{2}$-open and $\mathscr{T}_{2} \mathscr{T}_{1}$-open.

Definition 2.2. Let $\left(X, \mathscr{T}_{i}, \mathscr{T}_{j}\right), i, j=1,2 ; i \neq j$ be a bitopological space and $A, B \subseteq X$.

(i) $A$ is called $\mathscr{T}_{i} \mathscr{T}_{j}$-semiopen in $X$ provided there is a $\mathscr{T}_{i}$-open subset $O$ of $X$ such that $O \subseteq A \subseteq C l_{\widetilde{J_{j}}}(O)$, equivalently $A \subseteq C l_{\widetilde{J_{j}}}\left(\operatorname{Int}_{\widetilde{T_{i}}}(A)\right)$. It's complement is said to be $\mathscr{T}_{i} \cdot \mathscr{T}_{j}$-semiclosed [2].

(ii) The $\cdot \mathscr{T}_{i} \cdot T_{j}$-semiinterior of $A$ denoted by $\mathscr{T}_{i} \cdot T_{j}$ - $\operatorname{sint}(A)$ is defined as the union of $\mathscr{T}_{i} T_{j}$-semiopen subsets of $X$ contained in $A$. The $\mathscr{T}_{i} T_{j}$-semiclosure of $A$ denoted by $\mathscr{T}_{i} \mathscr{T}_{j}-s C l(A)$, is the intersection of all $\mathscr{T}_{i} \cdot \mathscr{T}_{j}$-semiclosed sets of $X$ containing $A$.

(iii) $A$ is said to be pairwise semiopen if it is both $\widetilde{T}_{1} \mathscr{T}_{2}$-semiopen and $\mathscr{T}_{2} \cdot \mathscr{T}_{1}$-semiopen [11].

(iv) $B$ is said to be a $\mathscr{T}_{i} \mathscr{T}_{j}$-semi-neighbourhood of $x \in X$ provided there is a $\mathscr{T}_{i} \mathscr{T}_{j}$-semiopen subset $O$ of $X$ such that $x \in O \subseteq B$.

The family of all $\widetilde{T}_{i} \cdot \widetilde{T}_{j}$-semiopen and $\mathscr{T}_{i} \cdot \mathscr{T}_{j}$-semiclosed subsets of $X$ are denote by $\mathscr{T}_{i} \cdot \mathscr{T}_{j} S O(X)$ and $\mathscr{T}_{i} \cdot \mathscr{T}_{j} S C(X)$ respectively.

Definition 2.3. [10] A point-to-set correspondence $F: X \rightarrow Y$ such that for each $x \in X, F(x)$ is a nonempty subset of $Y$ is said to be a multifunction .

Following Berge [5], the upper and lower inverse of $G \subseteq Y$ with respect to a multifunction $F$, are denoted and defined respectively by:

$$
F^{+}(G)=\{x \in X: F(x) \subset G\} \text { and } F^{-}(G)=\{x \in X: F(x) \cap G \neq \varnothing\} .
$$

Generally, for the lower inverse $F^{-}$between $Y$ and the power set $\mathscr{P}(X)$ of $X, F^{-}(y)=\{x \in X: y \in F(x)\}$ provided $y \in Y$. Clearly for $G \subseteq Y$, $F^{-}(G)=\bigcup\left\{F^{-}(y): y \in G\right\}$ and also,

$$
F^{+}(G)=X \backslash F^{-}(Y \backslash G) \text { and } F^{-}(G)=X \backslash F^{+}(Y \backslash G)
$$

For any $A \subseteq X, F(A)=\bigcup_{x \in A} F(x)$.

Definition 2.4. [10] A multifunction $F:\left(X, \mathscr{T}_{i}, \mathscr{T}_{j}\right) \rightarrow\left(Y, \mathscr{C}_{i}, \mathscr{Q}_{j}\right), i, j=1,2$; $i \neq j$ is said to be

(i) Upper $\mathscr{T}_{i} \mathscr{T}_{j}$-semi continuous at a point $x_{0} \in X$ provided for any $G_{1} \mathscr{C}_{2}$ open subset $V \subseteq Y$ such that $F\left(x_{o}\right) \subseteq V$, there exists an $\mathscr{T}_{i} \mathscr{T}_{j}$-semiopen set $U$ in $X$ with $x_{o} \in U$ such that $F(U) \subseteq V$ (or $U \subseteq F^{+}(V)$ ).

(ii) Lower $\mathscr{T}_{i} \cdot \mathscr{T}_{j}$-semi continuous at a point $x_{o} \in X$ provided for any $C_{i} C_{j}$ open set $V$ in $Y$ such that $F\left(x_{o}\right) \cap V \neq \varnothing$, there exists a $\mathscr{T}_{i} \mathscr{T}_{j}$-semiopen set $U$ in $X$ with $x_{o} \in U$ such that $F(z) \cap V \neq \varnothing$ for all $z \in U$ or $U \subseteq F^{-}(V)$.

(iii) Upper (resp Lower) $\mathscr{T}_{i} \cdot \mathscr{T}_{j}$-semi continuous if it is Upper (resp Lower) $\mathscr{T}_{i} \cdot \mathscr{T}_{j}$-semi continuous at all points of $X$.

Clearly, $F^{+}(V)$ and $F^{-}(V)$ are $\mathscr{T}_{i} \mathscr{T}_{j}$-open in $X$ for any $C_{i} C_{j}$-open set $V$ in $Y . F$ is $\mathscr{T}_{i} \mathscr{T}_{j}$-semicontinuous at $x_{o} \in X$ provided it is both upper and lower- $\mathscr{T}_{i} \cdot T_{j}$-continuous (resp., $\mathscr{T}_{i} \mathscr{T}_{j}$-semicontinuous) at $x_{o}$. Generally, $F$ 
is upper (resp lower)- $\widetilde{T}_{i} \cdot \widetilde{T}_{j}$-semi continuous if the multifunctions induced $F_{1}:\left(X, \mathscr{T}_{1}\right) \rightarrow\left(Y, \mathscr{C}_{1}\right)$ and $F_{2}:\left(X, \mathscr{T}_{2}\right) \rightarrow\left(Y, \mathscr{C}_{2}\right)$ are both upper (resp lower) semi continuous on $X$.

Definition 2.5. [10] [13] A subfamily $m_{X}$ of a power set $\mathscr{P}(X)$ of a nonempty set $X$ is said to be a minimal structure (briefly $m$-structure) on $X$ provided both $\varnothing$ and $X$ belongs to $m_{X}$. The pair $\left(X, m_{X}\right)$ is called an $m$-space and member of $\left(X, m_{X}\right)$ is said to be $m_{X}$-open.

Definition 2.6. [3] [13] A subset $A$ of $X$ in an $m$-space $\left(X, m_{X}\right)$ is said to be

(i) $m$-semiopen if $A \subseteq m C l(m \operatorname{Int}(A))$.

(ii) $m$-semiclosed if $\operatorname{mInt}(m C l(A)) \subseteq A$.

Definition 2.7. [10] For a minimal space $\left(X, m_{X}\right), m_{X}$ is said to have property ( $\mathscr{\beta})$ of Maki [3] if the union of any collection of $m_{X}$-open subsets of $X$ is also $m_{X}$-open.

Lemma 2.8. [13] For an $m$-space $\left(X, m_{X}\right)$, an arbitrary union of $m$-semiopen subsets of $X$ is also $m$-semiopen.

Lemma 2.9. (cf. [3] [10] [13]) For an $m$-space $\left(X, m_{X}\right)$ and subsets $A, B$ and $F$ of $X$ the properties below holds:

(i) $m_{X} s C l(X \backslash A)=X \backslash\left(m_{X} \operatorname{sint}(A)\right)$ and $m_{X} \operatorname{sint}(X \backslash A)=X \backslash(\operatorname{msCl}(A))$

(ii) $F$ is $m_{X}$-semiclosed if and only if $m_{X} \operatorname{scl}(F)=F$.

(iii) $A$ is $m_{X}$-semiopen if and only if $m_{X} \operatorname{sint}(A)=A$.

(iv) $m_{X} s C l(\varnothing)=\varnothing, m_{X} s C l(X)=X$ and $m_{X} \operatorname{sint}(\varnothing)=\varnothing$, $m_{X} \operatorname{sint}(X)=X$.

(v) If $A \subseteq B$, then $m_{X} s C l(A) \subseteq m_{X} s C l(B)$ and $m_{X} \operatorname{sint}(A) \subseteq m_{X} \operatorname{sint}(B)$

(vi) $m_{X} s C l\left(m_{X} s C l(A)\right)=m_{X} s C l(A)$ and $m_{X} \operatorname{sint}\left(m_{X} \operatorname{sint}(A)\right)=m_{X} \operatorname{sint}(A)$.

(vii) $A \subseteq m_{X} \operatorname{sCl}(A)$ and $m_{X} \operatorname{sint}(A) \subseteq A$.

Lemma 2.10. (cf. [3] [10] [13]) Let $\left(X, m_{X}\right)$ be an $m$-space and $A \subseteq X$. Then $x \in m_{X} \operatorname{sCl}(A)$ if and only if for every $m_{X}$-semiopen set $U$ containing $X$, $A \cap U \neq \varnothing$.

Lemma 2.11. [10] For an $m$-space $\left(X, m_{X}\right)$ and subsets $A$ and $F$ of $X$, the following properties are equivalent:

(i) $m_{X}$ has property $\mathscr{B}$,

(ii) $A \in m_{X}$ provided $m_{X} \operatorname{Int}(A)=A$,

(iii) $X \backslash F \in m_{X}$ provided $m_{X} C l(F)=F$.

Lemma 2.12. ([10]) Let $\left(X, m_{X}\right)$ be am $m$-space with $m_{X}$ satisfying property $D_{B}$ and $A \subseteq X$.The properties below holds:

(i) $m_{X} \operatorname{Int}(A)=A$ if and only if $A \in m_{X}$.

(ii) $m_{X} C l(A)=A$ if and only if $X \backslash A \in m_{X}$

(iii) $m_{X} \operatorname{Int}(A) \in m_{X}$ and $m_{X} C l(A)$ is $m_{X}$-closed.

\section{On m-Asymmetric Semi Open and Closed Sets}

This section introduces the notions of $m$-asymmetric semiopen and semiclosed sets in the framework of bitopological spaces satisfying certain minimal condi- 
tions.

Definition 3.1. Let $\left(X, \mathscr{T}_{i}, \mathscr{T}_{j}\right), i, j=1,2 ; i \neq j$ be a botopological space and $m_{X}$ a minimal structure on $X$ determined with respect to $m_{i}$ and $m_{j}$. Then an ordered pair $\left(\left(X, \widetilde{T}_{i}, \widetilde{T}_{j}\right), m_{X}\right)$ shall be called a minimal bitopological space (or $m_{i j}$-spaces).

Since in our sense the minimal structure $m_{X}$ on $X$ is determined by the left and right minimal structures $m_{i}$ and $m_{j}$ which are as a generalization of the two topologies $\mathscr{T}_{i}$ and $\mathscr{T}_{j}, i, j=1,2 ; i \neq j$, we shall denote it by $m_{i j}$ and call the pair $\left(\left(X, \mathscr{T}_{i}, \mathscr{T}_{j}\right), m_{i j}\right)$ (or $\left.\left(X, m_{i j}\right)\right)$ a minimal bitopological space unless explicitly stated.

Definition 3.2. Let $\left(X, m_{i j}\right), i, j=1,2 ; i \neq j$ be a minimal bitopological space and $A$ a subset of $X$. Then $\mathrm{A}$ is said to be $m_{i j}$-semiopen in $X$ if there exists an $m_{i}$-open set $U$ such that $U \subseteq A \subseteq C l_{m_{j}}(U)$. It is said to be $m_{i j}$-semiclosed in $X$ if there exists a $m_{i}$-open set $\mathrm{U}$ such that $C l_{m_{j}}(A) \subseteq U$ whenever $A \subseteq U$.

We shall denote the collection of all $m_{i j}$-semiopen and $m_{i j}$-semiclosed sets in $\left(X, m_{i j}\right)$ by $m_{i j} s O(X)$ and $m_{i j} s C(X)$ respectively.

Example 3.3. Let $X=\{-2,-1,0,1,2\}$, and the minimal structures $m_{1}$ and $m_{2}$ be define by $m_{1}=\{\varnothing,\{-1\},\{0\},\{0,1\},\{-2,0,1\}, X\}$ and $m_{2}=\{\varnothing,\{0\},\{2\},\{0,1\}, X\}$ respectively. The sets $\varnothing$ and $\{0\}$ are $m_{i j}$-open sets but $\{-1\}$ and $\{-1,1\}$ are not $m_{i j}$-open. Also, $\{1\}$ is neither $m_{i j}$-open nor $m_{i j}$-closed.

Remark 3.4. Let $\left(X, m_{i j}\right), i, j=1,2 ; i \neq j$ be a minimal bitopological space.

(i) if $m_{i}=\mathscr{T}_{i}$ and $m_{j}=\mathscr{T}_{j}$, the any $m_{i j}$-semiopen set is $\mathscr{T}_{i} \cdot \mathscr{T}_{j}$-semiopen.

(ii) every $m_{i j}$-open (resp. $m_{i j}$-closed) set is $m_{i j}$-semiopen (resp. $m_{i j}$-semiclosed), but the converse is not generally true as our next example illustrates.

Example 3.5. Let us define $m_{i}$ and $m_{2}$ on $X=\{-2,-1,0,1,2,3\}$ on $X$ by $m_{1}=\{\varnothing,\{-1\},\{0\},\{2\}, X\}$ and $m_{2}=\{\varnothing,\{-2\},\{-1\},\{-1,0\}, X\}$ respectively. Then the subset $A=\{-1,3\}$ of $X$ is $m_{i j}$-semiopen but not $m_{i j}$-open since $\{-1\} \subseteq A \subseteq C l_{m_{2}}(\{-1\})$, whence $A \notin m_{i j}$.

Proposition 3.6. Let $\left(X, m_{i j}\right), i, j=1,2 ; i \neq j$ be am $m_{i j}$-space and $A \subseteq X$. Then:

(i) $A \in m_{i j} s O(X)$ if and only if $A \subseteq C l_{m_{j}}\left(\operatorname{Int}_{m_{i}}(A)\right)$.

(ii) $A \in m_{i j} s C(X)$ if and only if $\operatorname{Int}_{m_{j}}\left(C l_{m_{i}}(A)\right) \subseteq A$.

Proof. (i) Suppose $A$ is a $m_{i j}$-semiopen subset of $X$. By Definition 3.2 we can always find an $m_{i}$-open subset $U$ of $X$ such that $U \subseteq A \subseteq C l_{m_{j}}(U)$. Since $U \subseteq \operatorname{Int}_{m_{i}}(A)$ and $U=\operatorname{Int}_{m_{i}}(U)$, we have

$$
A \subseteq C l_{m_{j}}(U) \subseteq C l_{m_{j}}\left(\operatorname{Int}_{m_{i}}(A)\right) \text {. }
$$

On the other hand, let $U=C l_{m_{j}}\left(\operatorname{Int}_{m_{i}}(A)\right)$, then, referring to Lemma 2.9, we have

$$
X \backslash U=X \backslash C l_{m_{j}}\left(\operatorname{Int}_{m_{i}}(A)\right)=\operatorname{Int}_{m_{j}}\left(X \backslash \operatorname{Int}_{m_{i}}(A)\right)=\operatorname{Int}_{m_{j}}\left(C l_{m_{i}}(X \backslash A)\right) .
$$

Thus, 


$$
C l_{m_{j}}(X \backslash U)=C l_{m_{j}}\left(\operatorname{Int}_{m_{j}}\left(C l_{m_{i}}(X \backslash A)\right)\right)=\operatorname{Int}_{m_{j}}\left(C l_{m_{i}}(X \backslash A)\right)=X \backslash U .
$$

Consequently, $X \backslash(X \backslash U) \in m_{i j} S O(X)$ and hence, $U \in m_{i j} s O(X)$. Therefore, $U \subseteq A \subseteq C l_{m_{j}}(U)$. This implies that $A$ is a $m_{i j}$-semiopen set.

(ii) Suppose $A$ is a $m_{i j}$-semiclosed set. By definition 3.2, there is some $m_{i}$-open set $U$ such that $C l_{m_{j}}(A) \subseteq U$. Since $C l_{m_{j}}(A) \subseteq C l_{m_{j}}(U)$ and $U=\operatorname{Int}_{m_{i}}(U)$, we have

$$
\operatorname{Int}_{m_{j}}\left(C l_{m_{i}}(A)\right) \subseteq \operatorname{Int}_{m_{j}}(A) \subseteq A
$$

and also from (i), $C l_{m_{j}}(A) \subseteq U$. This implies that $A$ is an $m_{i j}$-semiclosed set.

Generally, the $m_{i j}$-open sets and the $m_{i j}$-semiopen sets are not stable for the union. Nevertheless, for certain $m_{i j}$-structure, the class of $m_{i j}$-semiopen sets are stable under union of sets, like it is demonstrated in the following proposition.

Proposition 3.7. Let $\left(X, m_{i j}\right), i, j=1,2 ; i \neq j$ be an $m_{i j}$-space. Then, for a collection $\left\{A_{\gamma}: \gamma \in \Gamma\right\}$ of subsets of $X$, the following properties hold:

(i) $\bigcup_{\gamma \in \Gamma} A_{\gamma} \in m_{i j} s O(X)$ provided for all $\gamma \in \Gamma, A_{\gamma} \in m_{i j} s O(X)$.

(ii) $\bigcap_{\gamma \in \Gamma} A_{\gamma} \in m_{i j} s C(X)$ provided for all $\gamma \in \Gamma, A_{\gamma} \in m_{i j} s C(X)$.

Proof. (i) Suppose that $A_{\gamma} \in m_{i j} S O(X)$ for all $\gamma \in \Gamma$. Then, by definition 3.2 and Proposition 3.6, we have

$$
A_{\gamma} \subseteq C l_{m_{j}} \operatorname{Int}_{m_{i}}\left(A_{\gamma}\right) \subseteq C l_{m_{j}} \operatorname{Int}_{m_{i}}\left(\bigcup_{\gamma \in \Gamma} A_{\gamma}\right),
$$

which implies $\bigcup_{\gamma \in \Gamma} A_{\gamma} \subseteq C l_{m_{j}} \operatorname{Int}_{m_{i}}\left(\bigcup_{\gamma \in \Gamma} A_{\gamma}\right)$, so that, $\bigcup_{\gamma \in \Gamma} A_{\gamma} \in m_{i j} S O(X)$.

(ii) It follows from Proposition 3.7 (i) and De-Morgan's laws.

Remark 3.8. It should generally be noted that, the intersection of any two $m_{i j}$-semiopen sets may not be $m_{i j}$-semiopen in a minimal bitopological spaces $\left(X, m_{i j}\right)$, as illustrated in the next example.

Example 3.9. Define the minimal structures $m_{1}$ and $m_{2}$ on given set $X=\{-3,-2,-1,0,1,2,3\}$ by $m_{1}=\{\varnothing,\{0\},\{2\},\{0,2\}, X\}$ and $m_{2}=\{\varnothing,\{-2\},\{0\},\{2\},\{0,2\}, X\}$. We observer that, $\{-1,0\}$ and $\{-1,2\}$ are $m_{i j}$-semiopen sets, $i, j=1,2 ; i \neq j$. But then, $\{-1,0\} \cap\{-1,2\}=\{-1\}$ which is not $m_{i j}$ semiopen, indeed, $\{-1\} \nsubseteq C l_{m_{i}}\left(\operatorname{Int}_{m_{j}}(\{-1\})\right)=\varnothing$.

Definition 3.10. Let $\left(X, m_{i j}\right), i, j=1,2 ; i \neq j$ be an $m_{i j}$-space. A subset $O$ of $X$ is an $m_{i j}$-semineighborhood of a point $x \in X$ if there exists an $m_{i j}$-semiopen subset $U$ of $X$ such that $x \in U \subseteq O$.

Definition 3.11. Let $\left(X, m_{i j}\right), i, j=1,2 ; i \neq j$ be an $m_{i j}$-space and $A \subseteq X$. Then, the $m_{i j}$-semiinterior and $m_{i j}$-semiclosure of $A$ are respectively denoted and defined by:

(i) $m_{i j} \operatorname{sint}(A)=\bigcup\left\{U: U \subseteq A\right.$ and $\left.U \in m_{i j} S O(X)\right\}$,

(ii) $m_{i j} s C l(A)=\bigcap\left\{F: A \subseteq F\right.$ and $\left.F \in m_{i j} s C(X)\right\}$,

Remark 3.12. For any bitopological spaces $\left(X, \mathscr{T}_{1}, \mathscr{T}_{2}\right)$; 
(i) $\mathscr{T}_{i} \mathscr{T}_{j} S O(X)$ is a minimal structures of $X$.

(ii) In the following, we denote by $m_{i j}(X)$ a minimal structure on $X$ a generalization of $\mathscr{T}_{i}$ and $\mathscr{T}_{j}$. For $A \subseteq X$, if $m_{i j}(X)=\mathscr{T}_{i} \mathscr{T}_{j} S O(X)$, then by Definition 3.11;

(a) $m_{i j} \operatorname{Int}(A)=\mathscr{T}_{i} \cdot \mathscr{T}_{j} \operatorname{sint}(A)$,

(b) $m_{i j} C l(A)=\mathscr{T}_{i} \cdot \mathscr{T}_{j} s C l(A)$.

Proposition 3.13. For any $m_{i j}$-space $\left(X, m_{i j}\right), i, j=1,2 ; i \neq j$ and subsets $A$ and $B$ of $X$, the following $m_{i j}$-semiclosure and $m_{i j}$-semiinterior properties holds:

(i) $m_{i j} \operatorname{sint}(A) \subseteq A$ and $m_{i j} s C l(A) A \supseteq A$.

(ii) $m_{i j} \operatorname{sint}(A) \subseteq m_{i j} \operatorname{sint}(B)$ and $m_{i j} s C l(A) \subseteq m_{i j} s C(B)$ provided $A \subseteq B$.

(iii) $m_{i j} \operatorname{sint}(\varnothing)=\varnothing, m_{i j} \operatorname{sint}(X)=X, m_{i j} s C l(\varnothing)=\varnothing$ and $m_{i j} s C l(X)=X$.

(iv) $A=m_{i j} \operatorname{sint}(A)$ provided $A \in m_{i j} s O(X)$.

(v) $A=m_{i j} s C l(A)$ provided $X \backslash A \in m_{i j} s O(X)$.

(vi) $m_{i j} \operatorname{sint}\left(m_{i j} \operatorname{sint}(A)\right)=m_{i j} \operatorname{sint}(A)$ and $m_{i j} s C l\left(m_{i j} s C l(A)\right)=m_{i j} s C l(A)$

Proof. (i) and (ii) are obvious by the interior and closure property and using Proposition 3.6.

(iii) Follows from the interior and closure properties and also part (i) and (ii).

(iv) If $A \in m_{i j} S O(X)$, Definition 3.2 implies that, there exists an $m_{i}$-open subset $U$ of $X$ such that $U \subseteq A \subseteq C l_{m_{j}}(U)$. Since, $U \subseteq \operatorname{Int}_{m_{i}}(A)$ and $U=I n t_{m_{i}}(U)$, we obtain following Proposition 3.6 that

$$
A \subseteq C l_{m_{j}}(U) \subseteq C l_{m_{j}}\left(\operatorname{Int}_{m_{i}}(A)\right) .
$$

And also $A \supseteq C l_{m_{j}}\left(\operatorname{Int}_{m_{i}}(A)\right)$. Hence, $A=C l_{m_{j}}\left(\operatorname{Int}_{m_{i}}(A)\right)$

(v) Suppose $m_{i j} s C l(A)=A$. Then by Definition 3.11 and Proposition 3.7 we have

$$
X \backslash A=X \backslash m_{i j} \operatorname{sCl}(A)=m_{i j} \operatorname{sint}(X \backslash A)
$$

Hence by (i), $X \backslash A \in m_{i j} s O(X)$.

(vi) follows from (iii) and (iv).

Proposition 3.14. Let $\left(X, m_{i j}\right), i, j=1,2 ; i \neq j$ be an $m_{i j}$ space and $A \subseteq X$. Then, for each $U \in m_{i j} s O(X)$ such that $x \in U, U \cap A \neq \varnothing$ if and only if $x \in m_{i j} s C l(A)$.

Proof. Suppose that $x \in m_{i j} s C l(A)$. We need to show that for all $m_{i j}$-semiopen subset $U$ of $X$ containing $x, U \cap A \neq \varnothing$. On the contrary, suppose for some $m_{i j}$-semiopen set $U$ containing $x, U \cap A=\varnothing$. Then $A \subseteq X \backslash U$ and $X \backslash(X \backslash U) \in m_{i j} S O(X)$ and Proposition 3.13 implies $m_{i j} s C l(A) \subseteq m_{i j} s C l(X \backslash U)=X \backslash U$. Since $x \in m_{i j} s C l(A) \subseteq X \backslash U$, it follows that $x \notin U$ which contradicts our assertion. Consequently, $U \cap A \neq \varnothing$.

Conversely, suppose that for every $m_{i j}$-semiopen subset $U$ of $X$ containing $X$, $U \cap A \neq \varnothing$, then we need to show that $x \in m_{i j} \operatorname{sCl}(A)$. Suppose on the contrary, $x \notin m_{i j} s C l(A)$, then there exists an $m_{i j}$-semiclosed subset $F$ of $X$ such that $A \subseteq F$. Proposition 3.13 then implies $x \notin m_{i j} s C l(A) \subseteq m_{i j} s C l(F)=F$, so that 
$x \notin F$. Since $F \in m_{i j} s C(X)$, then $X \backslash F \in m_{i j} s O(X)$ and $x \in X \backslash F$ so that, $(X \backslash F) \cap A=\varnothing$. Setting $U=X \backslash F$, we obtaining $U \cap A=\varnothing$ which contradicts our assertion that $U \cap A \neq \varnothing$. Hence, $x \in m_{i i} s C l(A)$.

Proposition 3.15. For a minimal bitopological space $\left(X, m_{i j}\right), i, j=1,2$; $i \neq j$ and any $A \subseteq X$, the properties below holds:

(i) $m_{i j} s C l(X \backslash A)=X \backslash\left(m_{i j} \operatorname{sint}(A)\right)$,

(ii) $m_{i j} \operatorname{sint}(X \backslash A)=X \backslash\left(m_{i j} s C l(A)\right)$.

Proof.

(i) For each $A \subseteq X$ we obtain by Definition 3.11 and Proposition 3.13 that,

$$
\begin{aligned}
m_{i j} s C l(X \backslash A) & =\bigcap\left\{X \backslash V: X \backslash V \in m_{i j} s C(X), X \backslash A \subseteq X-V\right\} \\
& =\bigcap\left\{X \backslash V: V \in m_{i j} s O(X), V \subseteq A\right\} \\
& =X \backslash \bigcup\left\{V: V \subseteq A, V \in m_{i j} s O(X)\right\} \text { by De-Morgans Law } \\
& =X \backslash\left(m_{i j} \operatorname{sint}(A)\right)
\end{aligned}
$$

(ii) Let $x \in m_{i j} \operatorname{sint}(X \backslash A)$. Then, is some neighborhood $U_{x} \in m_{i j} s O(X)$ of $x$ for which $U \subseteq X \backslash A$. Because $x \notin A, U \cap A=\varnothing$. Proposition 3.14 then implies that, $x \notin m_{i j} s C l(A)$ and as a consequence, $x \in X \backslash m_{i j} s C l(A)$. Therefore,

$$
m_{i j} \operatorname{sint}(X \backslash A) \subseteq X \backslash\left(m_{i j} s C l(A)\right)
$$

Convesely, if $X \backslash m_{i j} s C l(A)=\varnothing$, the inclusion is obvious. Suppose $x \in X \backslash m_{i j} S C l(A)$, then by the complementation property $x \notin m_{i j} s C l(A)$ implying $x \notin A$. By Proposition 3.14, there exists an $m_{i j}$-semiopen neighborhood of $x, \quad V$ such that $U \cap A=\varnothing$. Thus, $V \subseteq X \backslash A$ giving $x \in m_{i j} s C l(X \backslash A)$. Hence,

$$
X \backslash\left(m_{i j} \operatorname{sint}(A)\right) \subseteq m_{i j} s C l(X \backslash A) .
$$

Proposition 3.16. For an $m_{i j}$ space $\left(X, m_{i j}\right), i, j=1,2 ; i \neq j$ and any subset $A \subseteq X$, the properties below are satisfied:

(i) $m_{i j} s C l(A)=\operatorname{Int}_{m_{j}}\left(C l_{m_{i}}(A)\right) \cup A$.

(ii) $m_{i j} S C l(A)=\operatorname{Int}_{m_{j}}\left(C l_{m_{i}}(A)\right)$ provided $A \in m_{i j} O(X)$. The converse to this assertion is not necessary true.

\section{Proof.}

(i) Since by Proposition 3.6, $m_{i j} s C l(A)$ is $m_{i j}$-semiclosed, we have

$$
m_{i j} s C l(A) \supseteq \operatorname{Int}_{m_{j}}\left(C l_{m_{i}}\left(m_{i j} s C l(A)\right)\right) .
$$

Thus, $\operatorname{Int}_{m_{i}}\left(C l_{m_{j}}(A)\right) \subseteq m_{i j} s C l(A)$. Since $A \subseteq m_{i j} s C l(A)$, it holds that,

$$
\operatorname{Int}_{m_{j}}\left(C l_{m_{i}}(A)\right) \cup A \subseteq m_{i j} s C l(A) \cup A=m_{i j} s C l(A) .
$$

Conversely, let $B=I n t_{m_{j}}\left(C l_{m_{i}}(A)\right) \cup A$. We aim to show that $\mathrm{B}$ is an $m_{i j^{-}}$ semiclosed set. By definition, 


$$
\begin{aligned}
\operatorname{Int}_{m_{j}}\left(C l_{m_{i}}(B)\right) & =\operatorname{Int}_{m_{j}}\left(C l_{m_{i}}\left(\operatorname{Int}_{m_{j}}\left(C l_{m_{i}}(A)\right) \cup A\right)\right) \\
& \subseteq \operatorname{Int}_{m_{j}}\left(C l_{m_{i}}\left(C l_{m_{i}}(A) \cup A\right)\right) \\
& =\operatorname{Int}_{m_{j}}\left(C l_{m_{i}}\left(C l_{m_{i}}(A)\right)\right) \\
& =\operatorname{Int}_{m_{j}}\left(C l_{m_{i}}(A)\right) \\
& \subseteq \operatorname{Int}_{m_{j}}\left(C l_{m_{i}}(A)\right) \cup A \\
& =B
\end{aligned}
$$

Thus, $B=\operatorname{Int}_{m_{j}}\left(C l_{m_{i}}(A)\right) \cup A$ is a $m_{i j}$-semiclosed set. Hence,

$$
\operatorname{Int}_{m_{j}}\left(C l_{m_{i}}(A)\right) \cup A \supseteq m_{i j} s C l(A) .
$$

Therefore, $m_{i j} s C l(A)=\operatorname{Int}_{m_{j}}\left(C l_{m_{i}}(A)\right) \cup A$.

(ii) Let $A$ be $m_{i j}$-open in $X$, then by Definition 3.2 and Proposition 3.6,

$$
A=\operatorname{Int}_{m_{j}}\left(\operatorname{Int}_{m_{i}}(A)\right) \subseteq \operatorname{Int}_{m_{j}}\left(C l_{m_{i}}(A)\right)
$$

Consequently, by (i),

$$
m_{i j} s C l(A)=\operatorname{Int}_{m_{j}}\left(C l_{m_{i}}(A)\right) .
$$

Example 3.17. In this example we aim to show that, the converse to part (ii) of Proposition 3.16 is not necessary true: Define the two minimal structures $m_{1}$ and $m_{2}$ on $X=\{-3,-2,-1,0,1,2\}$ by $m_{1}=\{\varnothing,\{-2,1\}, X\}$ and $m_{2}=\{\varnothing,\{-3\},\{2\}, X\}$. One clearly see that, $\{-2,1\}$ is only $m_{i}$-open but not $m_{i I}$-open even thought $m_{i j} s C l(\{-2,1\})=\operatorname{Int}_{m j}\left(C l_{m_{i}}(\{-2,1\})\right)$.

Remark 3.18. For a bitopological space $\left(X, \mathscr{T}_{i}, \mathscr{T}_{j}\right), i, j=1,2 ; i \neq j$ the families $\mathscr{T}_{i} \mathscr{T}_{j} O(X)$ and $m_{i j} s O(X)$ are all $m_{i j}$-structures of $X$ satisfying property $\mathscr{B}$.

Proposition 3.19. For an $m_{i j}$-space $\left(X, m_{i j}\right), i, j=1,2 ; i \neq j$ with $m_{i j}$ satisfying property ( $\mathscr{B}$ ) and subsets $A$ and $F$ of $X$, the properties below holds:

(i) $m_{i j} \operatorname{sint}(A)=A$ provided $A \in m_{i j} s O(X)$.

(ii) If $X \backslash F \in m_{i j} s O(X)$, then $m_{i j} s C l(F)=F$.

Proof. Suppose that $m_{i j}$ satisfying property ( $\mathscr{B}$ ):

(i) Let $A \in m_{i j} s O(X)$, then by Definition 3.11, $m_{i j} \operatorname{sint}(A) \in m_{i j} s O(X)$. Hence, $m_{i j} \operatorname{sint}(A)=A$.

(ii) If $m_{i j} s C l(F)=F$, then Definition 3.11 and Proposition 3.15 gives

$$
X \backslash F=X \backslash m_{i j} s C l(F)=m_{i j} \operatorname{sint}(X \backslash F)
$$

Thus, by part (i), we obtain $X \backslash F \in m_{i j} s O(X)$.

Proposition 3.20. For any $m_{i j}$-space $\left(X, m_{i j}\right), i, j=1,2 ; i \neq j$ with $m_{i j}$ satisfying property $\mathscr{R}$ and any $A \subseteq X$, the properties outlined below holds:

(i) $A=m_{i j} \operatorname{sint}(A)$ if and only if $A$ is $m_{i j}$-semiopen in $X$.

(ii) $A=m_{i j} s C l(A)$ if and only if $X \backslash A$ is $m_{i j}$-semiopen in $X$.

(iii) $m_{i j} \operatorname{sint}(A)$ is $m_{i j}$-semiopen.

(iv) $m_{i j} s C l(A)$ is $m_{i j}$-semiclosed.

Proof. (i) and (ii) follows $m_{i j}$-closed, $m_{i j}$ Interior, the property of $\mathscr{B}$ and 
also Proposition 3.13 and Proposition 3.19.

(iii) and (iv) follows from (i) and (ii) with the aid of Proposition 3.7, Proposition 3.13 and Proposition 3.19.

Proposition 3.21. Let $\left(X, m_{i j}\right), i, j=1,2 ; i \neq j$ be an $m_{i j}$ space with $m_{i j}$ satisfying the property $\mathscr{R}$ and let $\left\{A_{\gamma}: \gamma \in \Gamma\right\}$ be an arbitrary collection of subsets of $X$. Then, $\bigcup_{\gamma \in \Gamma} A_{\gamma} \in m_{i j} s O(X)$ provided $A_{\gamma} \subset m_{i j} s O(X)$ for every $\gamma \in \Gamma$.

Proof. Suppose that $m_{i j}$ satisfies property and $A_{\gamma} \in m_{i j} S O(X)$ for all $\gamma \in \Gamma$. By Definition 3.2, we can find some $m_{i}$-open set $U_{\gamma}$ such that $U_{\gamma} \subseteq A_{\gamma} \subseteq C l_{m_{j}} U_{\gamma}$ for some $\gamma \in \Gamma$. Consequently,

$$
\bigcup_{\gamma \in \Gamma}\left(U_{\gamma}\right) \subseteq \bigcup_{\gamma \in \Gamma}\left(A_{\gamma}\right) \subseteq \bigcup_{\gamma \in \Gamma}\left(C l_{m_{j}}\left(U_{\gamma}\right)\right)
$$

Since $C l_{m_{j}}$ is a monotone operator, $\bigcup_{\gamma \in \Gamma}\left(C l_{m_{j}}\left(U_{\gamma}\right)\right) \subseteq C l_{m_{j}}\left(\bigcup_{\gamma \in \Gamma}\left(U_{\gamma}\right)\right)$ and $\bigcup_{\gamma \in \Gamma}\left(U_{\gamma}\right)$ is $m_{i}$-open as $m_{i j}$ satisfies property $\mathscr{R}$. As a consequence, $\bigcup_{\gamma \in \Gamma}\left(U_{\gamma}\right) \subseteq \bigcup_{\gamma \in \Gamma}\left(A_{\gamma}\right) \subseteq C l_{m_{j}}\left(\bigcup_{\gamma \in \Gamma}\left(U_{\gamma}\right)\right)$. Therefore, $\bigcup_{\gamma \in \Gamma} A_{\gamma} \in m_{i j} s O(X)$.

Proposition 3.22. Let $\left(\left(X, \mathscr{T}_{i}, \mathscr{T}_{j}\right), m_{i j}\right), i, j=1,2 ; i \neq j$ be an $m_{i j}$-space and $A \subseteq X$. Provided $m_{i j}$ satisfy property $\mathscr{P}$, then:

(i) $m_{i j} s C l(A)=A \cup \operatorname{Int}_{m_{i}}\left(C l_{m_{i}}(A)\right)$, and

(ii) $m_{i j} \operatorname{sint}(A)=A \cap C l_{m_{i}}\left(\operatorname{Int}_{m_{i}}(A)\right)$ holds.

Proof. (i) Since $m_{i j}$ satisfy property $\mathscr{R}$, then $m_{i j} s C l(A)$ is an $m_{i j}$-semiclosed set. By Definition 3.2 and Proposition 3.6, we obtain that

$$
\operatorname{Int}_{m_{j}}\left(C l_{m_{i}}\left(m_{i j} s C l(A)\right)\right) \subseteq m_{i j} s C l(A) .
$$

Therefore, $\operatorname{Int}_{m_{j}}\left(C l_{m_{i}}(A)\right) \subseteq m_{i j} s C l(A)$ and it follows that, $A \cup \operatorname{Int}_{m_{j}}\left(C l_{m_{i}}(A)\right) \subseteq m_{i j} s C l(A)$.

On the other hand, we observe that

$$
\begin{aligned}
& \operatorname{Int}_{m_{j}}\left(C l_{m_{i}}\left(A \cup \operatorname{Int}_{m_{j}}\left(C l_{m_{i}}(A)\right)\right)\right) \\
& =\operatorname{Int}_{m_{i}}\left(C l_{m_{i}}(A)\right) \cup \operatorname{Int}_{m_{j}}\left(C l_{m_{i}}\left(\operatorname{Int}_{m_{j}}\left(C l_{m_{i}}(A)\right)\right)\right) \\
& \subseteq\left(C l_{m_{i}}(A)\right) \cup \operatorname{Int}_{m_{j}}\left(C l_{m_{i}}\left(\operatorname{Int}_{m_{j}}\left(C l_{m_{i}}(A)\right)\right)\right) \\
& =C l_{m_{i}}(A) \cup \operatorname{Int}_{m_{j}}\left(C l_{m_{i}}(A)\right) \\
& =C l_{m_{i}}(A) .
\end{aligned}
$$

Hence, Definition 3.2 and Proposition 3.6 implies,

$$
\operatorname{Int}_{m_{j}}\left(C l_{m_{i}}\left(A \cup \operatorname{Int}_{m_{j}}\left(C l_{m_{i}}(A)\right)\right)\right) \subseteq \operatorname{Int}_{m_{j}}\left(C l_{m_{i}}(A)\right) \subseteq A \cup \operatorname{Int}_{m_{j}}\left(C l_{m_{i}}(A)\right) .
$$

and so, 


$$
\operatorname{Int}_{m_{j}}\left(C l_{m_{i}}\left(A \cup \operatorname{Int}_{m_{j}}\left(C l_{m_{j}}(A)\right)\right)\right) \subseteq A \cup \operatorname{Int}_{m_{j}}\left(C l_{m_{j}}(A)\right) .
$$

Consequently, $A \cup \operatorname{Int}_{m_{j}}\left(C l_{m_{j}}(A)\right)$ is an $m_{i j}$ semiclosed set and so $m_{i j} s C l(A) \subseteq A \cup \operatorname{Int}_{m_{j}}\left(C l_{m_{j}}(A)\right)$. This completes the proof.

(ii) The proof follows from Theorems 3.13 and 3.20.

If the property $\mathscr{B}$ of Make is removed in the previous theorem, the equality does not necessarily hold as show in the example below:

Example 3.23. Defined two minimal structures $m_{1}$ and $m_{2}$ on $\mathbb{N}$ as: $m_{1}=\{\varnothing, P(\{2 n-1: n \in \mathbb{N}\}),\{2\}, \mathbb{N}\}$ and $m_{2}=\{\varnothing, P(\{2 n-1: n \in \mathbb{N}\}),\{2\}, \mathbb{N}\}$.

Then the collection of all $m_{i j}$-closed and $m_{i j}$-open subsets of $\mathbb{N}$ are:

$$
\begin{aligned}
& m_{i j} C(\mathbb{N})=\left\{\varnothing, P(\{2 n-1: n \in \mathbb{N}\})^{c}, \mathbb{N}-\{2\}, \mathbb{N}\right\} \\
& \text { and } m_{i j} O(\mathbb{N})=\{\varnothing, P(\{2 n-1: n \in \mathbb{N}\}),\{2\}, V, \mathbb{N}\},
\end{aligned}
$$

where $V \cap\{2 n-1: n \in \mathbb{N}\} \neq \varnothing$. Take $A=\{4\}$. Then $m_{i j} s C l(A)=\{4\}$, $m_{i j} C l(A)=\{2 n: n \in \mathbb{N}\}$ and $m_{i j} \operatorname{Int}(A)=\{2 n: n \in \mathbb{N}\}=\{2\}$. Further,

$$
A \bigcup \operatorname{int}_{m_{2}}\left(C l_{m_{i}}(A)\right)=\{2,4\} \text {. }
$$

Consequently, $m_{i j} s O(A) \subseteq A \bigcup \operatorname{int}_{m_{2}}\left(C l_{m_{i}}(A)\right)$.

\section{On M-Asymmetric Semi Continuous Multifunctions}

In this part of our paper, we introduce and investigate some properties of the notion of upper and lower $M$ asymmetric semi-continuous multifunctions.

Definition 4.1. Let $\left(\left(X, \mathscr{T}_{i}, \mathscr{T}_{j}\right), m_{i j}(X)\right)$ and $\left(\left(Y, \mathscr{T}_{1}, \mathscr{T}_{2}\right), m_{i j}(Y)\right)$, $i, j=1,2 ; i \neq j$ be minimal bitopological spaces. We shall call a multifunction $F:\left(\left(X, \mathscr{T}_{i}, \mathscr{T}_{j}\right), m_{i j}(X)\right) \rightarrow\left(\left(Y, \mathscr{C}_{j}, \mathscr{C}_{i}\right), m_{i j}(Y)\right)$ to be:

(i) Upper $m_{i j}(X)$-semi-continuous at some point $x_{o} \in X$ provided for any $m_{i j}(Y)$-open subset $V \subseteq Y$ satisfying $F\left(x_{o}\right) \subseteq V$, there is an $m_{i j}(X)$ -semiopen set $U$ in $X$ with $x_{o} \in U$ for which $F(U) \subseteq V$ (or $U \subseteq F^{+}(V)$ ).

(ii) Lower $m_{i j}(X)$-semi-continuous at some point $x_{o} \in X$ provided for each $m_{i j}(Y)$-open subset $V \subseteq Y$ satisfying $F\left(x_{o}\right) \cap V \neq \varnothing$, we can find an $m_{i j}(Y)$-semiopen set $U$ in $X$ with $x_{o} \in U$ such that for all $z \in U$, $F(z) \cap V \neq \varnothing$.

(iii) Upper (resp Lower) $m_{i j}(X)$-semi continuous if it is Upper (resp Lower) $m_{i j}(X)$-semi continuous at each and every point of $X$.

Example 4.2. Let us define the two minimal structures $m_{1}(X)$ and $m_{2}(X)$ on $X=\{-2,-1,0,1,2,3\}$ by $m_{1}(X)=\{\varnothing,\{-1\},\{2\},\{-1,2\}, X\}$ and $m_{2}(X)=\{\varnothing,\{-1\},\{2\},\{-1,2\}, X\}$. Also, let the minimal structure $m_{1}(Y)$ and $m_{2}(Y)$ on $Y=\{-3,-2,-1,0,1,2,3\}$ be defined by $m_{1}(Y)=\{\varnothing,\{-3\},\{-2,0,1,3\}, X\}$ and $m_{2}(Y)=\{\varnothing,\{-2\},\{0\},\{-2,0\},\{-2,0,1,3\}, X\}$. Defined a multifunction 


$$
\begin{aligned}
& F:\left(\left(X, \mathscr{T}_{1}, \mathscr{T}_{2}\right), m_{i j}\right) \rightarrow\left(\left(Y, \mathscr{C}_{1}, \mathscr{C}_{2}\right), m_{i j}\right) \text { by: } \\
& f(x)= \begin{cases}\{-2,0\} & x=-1 \\
\{-3,1,3\} & x=1 \\
\{-3,-2\} & x=2\end{cases}
\end{aligned}
$$

Clearly, $F$ is upper and lower $M$-asymmetric semi-continuous.

In this first part of these sections we discuss some characterizations of upper $m_{i j}$-semicontinuous multifunctions.

Theorem 4.3. Let $F:\left(\left(X, \mathscr{T}_{i}, \mathscr{T}_{j}\right), m_{i j}(X)\right) \rightarrow\left(\left(Y, \mathscr{C}_{j}, \mathscr{Q}_{i}\right), m_{i j}(Y)\right)$, $i, j=1,2 ; i \neq j$ be a multifunction where, $\left(\left(Y, \mathscr{Q}_{j}, \mathscr{Q}_{i}\right), m_{i j}(Y)\right)$ satisfies property $\mathscr{B} . F$ is upper $m_{i j}$-semicontinuous at some point $X$ of $X$ if and only if for every $m_{i j}(Y)$-open set $V$ in $Y$ with $F(x) \subseteq V, x \in m_{i j} \operatorname{sint}\left(F^{+}(V)\right)$.

Proof. Let $F$ be upper $m_{i j}$-semicontinuous at a point $x$ of $X$ and $V$ be an $m_{i j}(Y)$-open set such that $F(x) \subseteq V$. By Definition 4.1, there is some $m_{i j}(X)$ semiopen set $U$ containing $x$ for which $F(U) \subseteq V$. Therefore,

$x \in U \subseteq F^{+}(V)$. The $m_{i j}(X)$-semiopeness of $U$ implies, $U=m_{i j}(X) \operatorname{sint}(U)$. Because $\left(\left(Y, \mathscr{C}_{j}, \mathscr{C}_{i}\right), m_{i j}(Y)\right)$ satisfies property $\mathscr{B}$, we have from Definition 2.3 that, $x \in m_{i j}(X) \operatorname{sint}\left(F^{+}(V)\right)$.

Conversely, if $V$ is an $m_{i j}(Y)$-open set in $Y$ with $F(x) \subseteq V$ for which $x \in m_{i j}(X) \operatorname{sint}\left(F^{+}(V)\right)$, then by definition, there is some $m_{i j}(X)$-semiopen set $U$ in $X$ with $x \in U$ satisfying $U \subseteq F^{+}(V)$. This implies, $F(U) \subseteq V$ and so $F$ is upper $m_{i j}$-semicontinuous at a point $X$ of $X$.

Theorem 4.4. Provided $\left(\left(Y, Q_{j}, Q_{i}\right), m_{i j}(Y)\right), i, j=1,2 ; i \neq j$ satisfies property $\mathscr{B}$, the following properties are equivalent for a multifunction $F:\left(\left(X, \mathscr{T}_{i}, \mathscr{T}_{j}\right), m_{i j}(X)\right) \rightarrow\left(\left(Y, \mathscr{C}_{j}, \mathscr{C}_{i}\right), m_{i j}(Y)\right):$

(i) $F$ is upper $m_{i j}$-semicontinuous;

(ii) For every $m_{i j}(Y)$-open set $V$, the set $F^{+}(V)$ is $m_{i j}(X)$-semiopen;

(iii) For every $m_{i j}(Y)$-closed set $K$, the set $F^{-}(K)$ is $m_{i j}(X)$-semiclosed;

(iv) For every $m_{i j}(X)$-open set, the set inclusion $F\left(m_{i j}(X) s C l(A)\right) \subseteq m_{i j}(Y) C l(F(A))$ is true;

(v) For any subset $B$ of $Y$, the set inclusion $m_{i j}(X) s C l\left(F^{-}(B)\right) \subseteq F^{-}\left(m_{i j}(Y) C l(B)\right)$ holds true;

(vi) For any subset $B$ of $Y$, the set inclusion $F^{+}\left(m_{i j}(Y) \operatorname{Int}(B)\right) \subseteq m_{i j}(X) \operatorname{sint}\left(F^{+}(B)\right)$ holds.

Proof. (i) $\Rightarrow$ (ii): let $V$ be a $m_{i j}(Y)$-open set of $Y$ and $x \in F^{+}(V)$ for all $x \in X$ then, $F(x) \subseteq V$. From the hypothesis, we can find an $m_{i j}(X)$-semi-open set $U$ with $x \in U$ for which $F(U) \subseteq V$. And so, one gets $x \in U \subseteq F^{+}(V)$ for every $x$ belongning to $F^{+}(V)$. Consequently by Proposition 3.6, 


$$
x \in U \subseteq C l_{m_{j}}\left(\operatorname{Int}_{m_{i}}(U)\right) \subseteq C l_{m_{j}}\left(\operatorname{Int}_{m_{i}}\left(F^{+}(V)\right)\right) .
$$

This implies that $F^{+}(V) \subseteq m_{i j}(X) \operatorname{sint}\left(F^{+}(V)\right)$. By Proposition 3.13, we have that, $F^{+}(V) \supseteq m_{i j} \operatorname{sint}\left(F^{+}(V)\right)$. This gives, $F^{+}(V)=m_{i j} \operatorname{sint}\left(F^{+}(V)\right)$ and so, $F^{+}(V)$ is $m_{i j}(X)$ semi-open in $X$.

(ii) $\Rightarrow$ (iii): Let $K \subset m_{i j} S C(Y)$. Since $Y \backslash K \in m_{i j}(Y) O(Y)$ then, we have from $F^{+}(Y \backslash K)=X \backslash F^{-}(K)$, Proposition 3.13 and Proposition 3.19 that,

$$
\begin{aligned}
& X \backslash F^{-}(K)=F^{+}(Y \backslash K)=m_{i j}(X) \operatorname{sint}\left(F^{+}(Y \backslash K)\right) \\
&=m_{i j}(X) \operatorname{sint}\left(X \backslash F^{-}(K)\right) \\
&=X \backslash m_{i j}(X) \operatorname{sCl}\left(F^{-}(K)\right) \\
& X \backslash m_{i j}(X) \operatorname{sCl}\left(F^{-}(K)\right)=m_{i j}(X) \operatorname{sint}\left(X \backslash F^{-}(K)\right) \\
&=m_{i j}(X) \operatorname{sint}\left(F^{+}(Y \backslash K)\right) \\
&=F^{+}(Y \backslash K) \\
&=X \backslash F^{-}(K)
\end{aligned}
$$

Hence,

$$
F^{-}(K)=m_{i j}(X) s C l\left(F^{-}(K)\right),
$$

implying $F^{-}(K)$ is $m_{i j}(X)$-semiclosed.

(iii) $\Rightarrow$ (iv): If $A$ is some subset of $X$, Definition 3.11 implies,

$$
\begin{aligned}
& F^{-}\left(m_{i j}(Y) C l(F(A))\right) \\
& =F^{-}\left(\bigcap\left\{K \subseteq Y: F(A) \subseteq K \text { and } K \in m_{i j} C(Y)\right\}\right) \\
& \supseteq \bigcap\left\{F^{-}(K) \subseteq X: A \subseteq F^{-}(K) \text { and } F^{-}(K) \in m_{i j} s C(X)\right\} \\
& =\bigcap\left\{L \subseteq X: A \subseteq L \text { and } L \in m_{i j} s C(X)\right\} \\
& =m_{i j}(X) s C l(A) .
\end{aligned}
$$

And so, $m_{i j}(Y) C l(F(A)) \supseteq F\left(m_{i j}(X) s C l(A)\right)$.

(iv) $\Rightarrow$ (v): Proposition 3.13 and Proposition 3.20 implies $m_{i j}(Y) C l(B)$ is a $m_{i j}(Y)$-closed set for if $B$ is any subset of $Y$. Proposition 3.13 and (iv) of this theorem gives:

$$
\begin{aligned}
& F^{-}\left(m_{i j}(Y) C l(B)\right) \\
& =F^{-}\left(\bigcap\left\{Q \subseteq Y: B \subseteq Q \text { and } Q \in m_{i j} C(Y)\right\}\right) \\
& =\bigcap\left\{F^{-}(Q) \subseteq X: F^{-}(B) \subseteq F^{-}(Q) \text { and } F^{-}(Q) \in m_{i j} s C(X)\right\} \\
& \supseteq \bigcap\left\{R \subseteq X: F^{-}(B) \subseteq R \text { and } R \in m_{i j} s C(X)\right\} \\
& =m_{i j}(X) s C l\left(F^{-}(B)\right) .
\end{aligned}
$$

and the implication follows.

(v) $\Rightarrow$ (vi): Using Definition 2.3, $m_{i j}(Y) \operatorname{Int}(B)=Y \backslash m_{i j}(Y) C l(Y \backslash B)$, Proposition 3.13 and (v), it follows for a subset $B$ of $Y$ that, 


$$
\begin{aligned}
X \backslash m_{i j}(X) \operatorname{sint}\left(F^{+}(B)\right) & =m_{i j}(X) s C l\left(X \backslash F^{+}(B)\right) \\
& =m_{i j}(X) s C l\left(F^{-}(Y \backslash B)\right) \\
& \subseteq F^{-}\left(m_{i j}(Y) C l(Y \backslash B)\right) \\
& =F^{-}\left(Y \backslash m_{i j}(Y) \operatorname{Int}(B)\right) \\
& =X \backslash F^{+}\left(m_{i j}(Y) \operatorname{Int}(B)\right) .
\end{aligned}
$$

Consequently, $F^{+}\left(m_{i j}(Y) \operatorname{Int}(B)\right) \subseteq m_{i j}(X) \operatorname{sint}\left(F^{+}(B)\right)$.

(vi) $\Rightarrow$ (ii): Since $Y \backslash V$ is a $m_{i j}(Y)$-closed set for any $m_{i j}(Y)$-open set $V$ in $Y$, (vi) and Proposition 3.13 implies;

$$
\begin{aligned}
X \backslash F^{+}(V) & \supseteq X \backslash F^{+}\left(m_{i j}(Y) \operatorname{Int}(V)\right) \\
& =F^{-}\left(Y \backslash m_{i j}(Y) \operatorname{Int}(V)\right) \\
& =F^{-}\left(m_{i j}(Y) C l(Y \backslash V)\right) \\
& \supseteq m_{i j}(X) s C l\left(F^{-}(Y \backslash V)\right) \quad \text { by (iv) } \\
& =m_{i j}(X) s C l\left(X \backslash F^{+}(V)\right) \\
& =X \backslash m_{i j}(X) \operatorname{sint}\left(F^{+}(V)\right) .
\end{aligned}
$$

Consequently, we obtain

$F^{+}(V) \subseteq F^{+}\left(m_{i j}(Y) \operatorname{Int}(V)\right) \subseteq m_{i j}(X) \operatorname{sint}\left(F^{+}(V)\right)$, implying $F^{+}(V)$ is an $m_{i j}(X)$-semiopen set in $X$.

(ii) $\Rightarrow$ (i): For any point $x \in X$ and $m_{i j}(Y)$-open set $V$ in $Y$ such that $F^{+}(x) \subseteq V$, part (ii) of this theorem implies, $F^{+}(V)$ is a $m_{i j}(X)$-semiopen set in $X$ with $x \in F^{+}(V)$. Setting $x \in F^{+}(V)$, we have by the $F(U) \subseteq V$ and consequently, by Definition 4.1, $F$ is upper $m_{i j}$-semicontinuous.

This second part of the sections gives some characterizations of lower $m_{i j}$-semicontinuous multifunctions.

Theorem 4.5. Provided $\left(\left(Y, C_{j}, Q_{i}\right), m_{i j}(Y)\right) i, j=1,2 ; i \neq j$, satisfies property $\mathscr{R}$, a multifunction $F:\left(\left(X, \mathscr{T}_{i}, \mathscr{T}_{j}\right), m_{i j}(X)\right) \rightarrow\left(\left(Y, \mathscr{C}_{j}, \mathscr{C}_{i}\right), m_{i j}(Y)\right)$ is lower $m_{i j}$-semicontinuous at $x \in X$ if and only if $x \in m_{i j} \operatorname{sint}\left(F^{-}(V)\right)$ for every $m_{i j}(Y)$-open set $V$ of $Y$ such that $F(x) \cap V \neq \varnothing$.

Proof. Suppose $F$ is lower $m_{i j}$-semicontinuous at $x_{o} \in X$. Let $V$ be an $m_{i j}(Y)$-open set satisfying $F(x) \cap V \neq \varnothing$ for $x_{o} \in X$. By Definition 4.1, there is an $m_{i j}(X)$-semiopen set $U$ with $x_{o} \in U$ such that $F(x) \cap V \neq \varnothing$ for all $x \in U$. Thus, from Definition 2.3, $x \in U \subseteq F^{-}(V)$. Because $U$ is $m_{i j}(X)$ -semiopen, $U=m_{i j}(X) \operatorname{sint}(U)$. Since $\left(\left(Y, \mathscr{Q}_{j}, \mathscr{Q}_{i}\right), m_{i j}(Y)\right)$ satisfies property $\mathscr{B}$, we get

$$
x \in m_{i j}(X) \operatorname{sint}(U) \subseteq m_{i j}(X) \operatorname{sint}\left(F^{+}(V)\right) .
$$

Conversely, suppose $V$ is an $m_{i j}(Y)$-open set with $F(x) \cap V \neq \varnothing$. From the assumption, $x \in m_{i j}(X) \operatorname{sint}\left(F^{+}(V)\right)$. By Definition 2.3 and 4.1, there exists a $m_{i j}(X)$-semiopen set $O$ in $X$ containing $X$ such that for all $z \in O, F(z) \cap V \neq \varnothing$. Clearly, $O \subseteq F^{-}(V)$ so that, $F(O) \subseteq V$ by Definition 2.3. Consequently, $F$ is 
lower $m_{i j}$-semicontinuous at a point $x$ of $X$.

Theorem 4.6. If $\left(\left(Y, Q_{j}, Q_{i}\right), m_{i j}(Y)\right), i, j=1,2 ; i \neq j$, satisfies property $\mathscr{R}$, then the following properties are equivalent for a multifunction $F:\left(\left(X, \mathscr{T}_{i}, \mathscr{T}_{j}\right), m_{i j}(X)\right) \rightarrow\left(\left(Y, \mathscr{Q}_{j}, \mathscr{Q}_{i}\right), m_{i j}(Y)\right):$

(i) $F$ is lower $m_{i j}$-semicontinuous;

(ii) For any $m_{i j}(Y)$-open set $V$ in $Y$, the set $F^{-}(V)$ is $m_{i j}(X)$-semiopen in $X$

(iii) For any $m_{i j}(Y)$-closed set $K$ in $Y$, the set $F^{+}(K)$ is $m_{i j}(X)$-semiclosed in $X$;

(iv)Given any subset $B$ of $Y, m_{i j}(X) s C l\left(F^{+}(B)\right) \subseteq F^{+}\left(m_{i j}(Y) C l(B)\right)$;

(v) Given any every $A \in m_{i j}(X), F\left(m_{i j} s C l(A)\right) \subseteq C l(F(A))$;

(vi) Given any subset $B \in Y$, the inclusion $F^{-}\left(m_{i j}(Y) \operatorname{Int}(B)\right) \subseteq m_{i j}(X) \operatorname{sint}\left(F^{-}(B)\right)$ holds true.

Proof. (i) $\Rightarrow$ (ii): let $V$ be a $m_{i j}(Y)$-open set of $Y$ such that $F^{-}(x) \cap V \neq \varnothing$ for a point $x \in X$. By the hypothesis, we can find an $m_{i j}(X)$-semiopen set $O$ with $x \in O$ such that for any $z \in O, F(z) \cap V \neq \varnothing$ holds. Since $O$ is $m_{i j}(X)$ -semiopen, $O=m_{i j}(X) \operatorname{sint}(U)$. Because $\left(\left(Y, Q_{j}, Q_{i}\right), m_{i j}(Y)\right)$ satisfies property $\mathscr{R}$, we then obtain;

$$
x \in m_{i j}(X) \operatorname{sint}(U) \subseteq m_{i j}(X) \operatorname{sint}\left(F^{-}(V)\right),
$$

implies $F^{-}(V) \subseteq m_{i j}(X) \operatorname{sint}\left(F^{-}(V)\right)$. By Proposition 3.13, $F^{-}(V) \supseteq m_{i j} \operatorname{sint}\left(F^{-}(V)\right)$ and so, $F^{-}(V)$ is $m_{i j}(X)$ semiopen.

(ii) $\Rightarrow$ (iii): If $K$ is any $m_{i j}(Y)$-closedset in $Y$ then, $Y-K \in m_{i j}(Y) O(Y)$. Thus,

$$
\begin{aligned}
X \backslash F^{+}(K) & =F^{-}(Y \backslash K)=m_{i j}(X) \operatorname{sint}\left(F^{-}(Y \backslash K)\right) \\
& =m_{i j}(X) \operatorname{sint}\left(X \backslash F^{+}(K)\right) \\
& =X \backslash m_{i j}(X) s C l\left(F^{+}(K)\right) .
\end{aligned}
$$

Which shows that, $F^{+}(K)=m_{i j}(X) s C l\left(F^{+}(K)\right)$ implying $F^{+}(K)$ is $m_{i j}(X)$-semiclosed.

(iii) $\Rightarrow$ (iv): By Proposition 3.13 and Proposition 3.20, $m_{i j}(Y) C l(B)$ is $m_{i j}(Y)$-closed in $Y$ for any subset $B$ of $Y$. From (iii), we obtain that

$$
\begin{aligned}
& m_{i j}(X) s C l\left(F^{+}(B)\right) \\
& =\bigcap\left\{F^{+}(K) \subseteq X: F^{+}(B) \subseteq F^{+}(K) \text { and } F^{+}(K) \in m_{i j} s C(X)\right\} \\
& \subseteq F^{+}\left(\bigcap\left\{K \subseteq Y: B \subseteq K \text { and } K \in m_{i j} C(Y)\right\}\right) \\
& =F^{+}\left(m_{i j}(Y) C l(B)\right)
\end{aligned}
$$

Thus, $m_{i j}(X) s C l\left(F^{+}(B)\right) \subseteq F^{+}\left(m_{i j}(Y) C l(B)\right)$. 
(iv) $\Rightarrow$ (v): For $A \subseteq X$ we have for (iv) that,

$$
\begin{aligned}
& F^{+}\left(m_{i j}(Y) C l(F(A))\right) \\
& =F^{+}\left(\bigcap\left\{K \subseteq Y: F(A) \subseteq K \text { and } K \in m_{i j} C(Y)\right\}\right) \\
& =\bigcap\left\{F^{+}(K) \subseteq X: A \subseteq F^{+}(K) \text { and } F^{+}(K) \in m_{i j} s C(X)\right\} \\
& \supseteq \bigcap\left\{D \subseteq X: A \subseteq D \text { and } D \in m_{i j} s C(X)\right\} \\
& =m_{i j}(X) s C l(A) .
\end{aligned}
$$

Consequently, $F\left(m_{i j}(X) s C l(A)\right) \subseteq m_{i j}(Y) C l(F(A))$.

(v) $\Rightarrow$ (vi): Because $m_{i j}(Y) \operatorname{Int}(B)=Y \backslash m_{i j}(Y) C l(Y \backslash B)$ for a set $B \subseteq Y$, Proposition 3.13 and part (v), then implies:

$$
\begin{aligned}
F^{-}\left(m_{i j}(Y) \operatorname{Int}(B)\right) & =F^{-}\left(Y \backslash m_{i j}(Y) C l(Y \backslash B)\right) \\
& =X \backslash F^{+}\left(m_{i j}(Y) C l(Y \backslash B)\right) \\
& \subseteq X \backslash m_{i j}(X) s C l\left(F^{+}(Y \backslash B)\right) \\
& =X \backslash m_{i j}(X) s C l\left(X \backslash F^{-}(B)\right) \\
& =m_{i j}(X) \operatorname{sint}\left(F^{-}(B)\right) .
\end{aligned}
$$

Consequently, $F^{+}\left(m_{i j}(Y) \operatorname{Int}(B)\right) \subseteq m_{i j}(X) \operatorname{sint}\left(F^{+}(B)\right)$.

(vi) $\Rightarrow$ (i): From (vi), we obtain for $x \in X$ and $m_{i j}(Y)$-open set $V$ in $Y$ satisfying $F^{+}(x) \cap V \neq \varnothing$ that,

$$
x \in F^{-}(V)=F^{-}\left(m_{i j}(Y) \operatorname{Int}(V)\right) \subseteq m_{i j}(X) \operatorname{sint}\left(F^{-}(V)\right) .
$$

Thus, there is an $m_{i j}(X)$-semiopen set $O$ with $x \in O$ for which $F^{+}(z) \cap V \neq \varnothing$ for all $z \in O$. This implies $F^{-}(V)$ is $m_{i j}(X)$-semiopen and so, $F$ is a lower $m_{i j}$-semicontinuous multifunction.

Lemma 4.7. For a minimal bitopological space $\left(\left(X, \mathscr{T}_{i}, \mathscr{T}_{j}\right), m_{i j}(X)\right)$, $i, j=1,2 ; i \neq j$, and for a subset $A$ of $X$, the properties below holds:

(i) $C l_{m_{j}}\left(\operatorname{Int}_{m_{i}}(A)\right) \subseteq C l_{m_{j}}\left(\operatorname{Int}_{m_{i}}\left(m_{i j} s C l(A)\right)\right) \subseteq m_{i j} s C l(A)$.

(ii) $m_{i j} \operatorname{sint}(A) \subseteq \operatorname{Int}_{m_{j}}\left(C l_{m_{i}}\left(m_{i j} \operatorname{sInt}(A)\right)\right) \subseteq \operatorname{Int}_{m_{j}}\left(C l_{m_{i}}(A)\right)$.

Proof. (i) For $A \subseteq X$, Proposition 3.13, $m_{i j} s C l(A)$ is an $m_{i j}$-semiclosed set. Hence from Proposition 3.6, we have

$$
C l_{m_{j}}\left(\operatorname{Int}_{m_{i}}(A)\right) \subseteq C l_{m_{j}}\left(\operatorname{Int}_{m_{i}}\left(m_{i j} s C l(A)\right)\right) \subseteq m_{i j} s C l(A) .
$$

(ii) Similarly, by Proposition 3.13, $m_{i j} \operatorname{sint}(A)$ is an $m_{i j}$-semiopen set and by Proposition 3.6, we have

$$
m_{i j} \operatorname{sint}(A) \subseteq \operatorname{Int}_{m_{j}}\left(C l_{m_{i}}\left(m_{i j} \operatorname{sint}(A)\right)\right) \subseteq \operatorname{Int}_{m_{j}}\left(C l_{m_{i}}(A)\right) .
$$

The concepts of Theorem 4.4 and 4.6 and, Lemma 4.7, leads us the theorem that follows:

Theorem 4.8. For a multifunction

$F:\left(\left(X, \mathscr{T}_{i}, \mathscr{T}_{j}\right), m_{i j}(X)\right) \rightarrow\left(\left(Y, \mathscr{Q}_{j}, \mathscr{Q}_{i}\right), m_{i j}(Y)\right), i, j=1,2 ; i \neq j$ with $\left(\left(Y, Q_{j}, Q_{i}\right), m_{i j}(Y)\right)$ satisfying property $\mathscr{R}$, the statements below are equiva- 
lent:

(i) $F$ is lower $m_{i j}$ semicontinuous;

(ii) $F^{-}(V) \subseteq C l_{m_{j}}\left(\operatorname{Int}_{m_{i}}\left(F^{-}(V)\right)\right)$ for any $m_{i j}(Y)$-open set $V$ in $Y$,

(iii) $\operatorname{Int}_{m_{j}}\left(C l_{m_{i}}\left(F^{-}(K)\right)\right) \subseteq F^{-}(K)$ for any $m_{i j}(Y)$-closed set $K$ in $Y$,

(iv) For $A \in X, F\left(\operatorname{Int}_{m_{j}}\left(C l_{m_{i}}(A)\right)\right) \subseteq m_{i j}(Y) C l(F(A))$;

(v) $\operatorname{Int}_{m_{j}}\left(C l_{m_{i}}\left(F^{-}(B)\right)\right) \subseteq F^{-}\left(m_{i j}(Y) C l(B)\right)$ for any subset $B$ of $Y$;

(vi) $F^{-}\left(m_{i j}(Y) \operatorname{Int}(B)\right) \subseteq C l_{m_{j}}\left(\operatorname{Int}_{m_{j}}\left(F^{-}(B)\right)\right)$ for any subset $B$ of $Y$.

Proof. (i) $\Rightarrow$ (ii): From Theorem 4.6 and Proposition 3.6, we obtain

$$
x \in m_{i j}(X) \operatorname{sint}(U) \subseteq m_{i j}(X) \operatorname{sint}\left(F^{-}(V)\right) .
$$

Consequently, $F^{-}(V) \subseteq C l_{m_{j}}\left(\operatorname{Int}_{m_{i}}\left(F^{-}(V)\right)\right)$.

(ii) $\Rightarrow$ (iii): If $K$ is a $m_{i j}(Y)$-closed subset in $Y$, then by Theorem 4.4 and Proposition 3.6,

$$
\begin{aligned}
X \backslash F^{-}(K) & =F^{+}(Y \backslash K) \subseteq m_{i j}(X) \operatorname{sInt}\left(F^{+}(Y \backslash K)\right) \\
& =m_{i j}(X) \operatorname{sint}\left(X \backslash F^{-}(K)\right) \\
& =X \backslash m_{i j}(X) s C l\left(F^{-}(K)\right) .
\end{aligned}
$$

Consequently, $F^{-}(K) \supseteq C l_{m_{j}}\left(\operatorname{Int}_{m_{i}}\left(F^{-}(K)\right)\right)$.

(iii) $\Rightarrow$ (iv): By Theorem 4.4 and Lemma 4.7, we obtain for a subset $A$ of $X$ that,

$$
\operatorname{Int}_{m_{j}}\left(C l_{m_{i}}(A)\right) \subseteq m_{i j}(X) s C l(A) \subseteq F^{-}\left(C l_{m_{i}}(F(A))\right)
$$

This shows that, $F\left(\operatorname{Int}_{m_{j}}\left(C l_{m_{i}}(A)\right)\right) \subseteq C l_{m_{i}}(F(A))$.

(iv) $\Rightarrow$ (v): From (iv) and Proposition 3.13, we obtain for any $B \subseteq Y$ that,

$$
\begin{aligned}
& F^{-}\left(m_{i j}(Y) C l(B)\right) \\
& =F^{-}\left(\bigcap\left\{K \subseteq Y: B \subseteq K \text { and } K \in m_{i j} C(Y)\right\}\right) \\
& =\bigcap\left\{F^{-}(K) \subseteq X: F^{-}(B) \subseteq F^{-}(K) \text { and } F^{-}(K) \in m_{i j} s C(X)\right\} \\
& \supseteq \bigcap\left\{Q \subseteq X: F^{-}(B) \subseteq Q \text { and } Q \in m_{i j} s C(X)\right\} \\
& =m_{i j}(X) s C l\left(F^{-}(B)\right) .
\end{aligned}
$$

By Proposition 3.6, $\operatorname{Int}_{m_{j}}\left(C l_{m_{i j}}\left(F^{-}(B)\right)\right) \subseteq F^{-}\left(m_{i j}(Y) C l(B)\right)$.

(v) $\Rightarrow$ (vi): From Proposition 3.13 and (v), one obtain

$$
\begin{aligned}
F^{-}\left(m_{i j}(Y) \operatorname{Int}(B)\right) & =F^{-}\left(Y \backslash m_{i j}(Y) C l(Y \backslash B)\right) \\
& =X \backslash F^{+}\left(m_{i j}(Y) C l(Y \backslash B)\right) \\
& \subseteq X \backslash m_{i j}(X) s C l\left(F^{+}(Y \backslash B)\right) \\
& =X \backslash m_{i j}(X) s C l\left(X \backslash F^{-}(B)\right) \\
& =m_{i j}(X) \operatorname{sint}\left(F^{-}(B)\right) .
\end{aligned}
$$

Thus, $F^{-}\left(m_{i j}(Y) \operatorname{Int}(B)\right) \subseteq C l_{m_{j}}\left(\operatorname{Int}_{m_{j}}\left(F^{-}(B)\right)\right)$. 
(vi) $\Rightarrow$ (i): For an $m_{i j}(Y)$-open set $V$ in $Y$ we obtain from (vi) and Proposition 3.13 that,

$$
F^{-}(V)=F^{-}\left(m_{i j}(Y) \operatorname{Int}(V)\right) \subseteq C l_{m_{j}}\left(\operatorname{Int}_{m_{i}}\left(F^{-}(V)\right)\right) .
$$

As such, $F^{-}(V)$ is $m_{i j}(X)$-semiopen in $X$ and by (ii), $F$ is lower $m_{i j-}$-semicontinuous.

\section{Conclusion}

In the first part of this paper, we have investigated and then generalized Maki [3], Prasad [2] and Bose [4] extensions of the weak forms of sets: the semiopen sets, to asymmetric topologies with sets satisfying certain minimal conditions. In the second part of our work, we have to use Noiri and Popa's [10] ideas to successfully introduce and investigate several fundamental properties of upper and lower $M$-asymmetric semicontinuous multifunctions defined between sets satisfying some minimal conditions in the realm of bitopological spaces. We have observed that the $M$-asymmetric semicontinuous multifunctions have properties similar to those of upper and lower continuous functions and $M$-continuous multifunctions between topological spaces, with the only difference that, the semiopen sets use in our results belonging to two topologies.

\section{Acknowledgements}

The authors wish to acknowledge the support of Mulungushi University and the refereed authors for their helpful work towards this paper. They also thank the anonymous referees for their helpful comments towards the improvement of the paper.

\section{Conflicts of Interest}

The authors declare no conflicts of interest regarding the publication of this paper.

\section{References}

[1] Levine, N. (1963) Semi-Open Sets and Semi-Continuity in Topological Spaces. The American Mathematical Monthly, 70, 36-41. https://doi.org/10.1080/00029890.1963.11990039

[2] Maheshwari, S.N. and Prasad, R. (1977) Semi-Open Sets and Semi-Continuous Function in Bitopological Spaces. Mathematical Notes, 26, 29-37.

[3] Maki, H., Rao, K.C. and Gani, A.N. (1999) On Generalizing Semi-Open Sets and Preopen Sets. Pure and Applied Mathematika Sciences, 49, 17-30.

[4] Bose, S. (1981) Semi Open Sets, Semi Continuity and Semi Open Mappings in Bitopological Spaces. Bulletin of the Calcutta Mathematical Society, 73, 237-246.

[5] Berge, C. (1997) Topological Spaces: Including a Treatment of Multi-Valued Functions, Vector Spaces and Convexity. Oliver and Boyd Ltd., Edinburgh.

[6] Whyburn, G.T. (1965) Continuity of Multifunctions. Proceedings of the National Academy of Sciences of the United States of America, 54, 1494-1501. 
https://doi.org/10.1073/pnas.54.6.1494

[7] Smithson, E.R. (1971) Multifunctions and Bitopological Spaces. Journal of Natural Sciences and Mathematics, XI, 191-198.

[8] Popa, V. (1975) Multifunctions and Bitopological Spaces. Bulletin mathématique de la Société des Sciences Mathématiques de la République Socialiste de Roumanie, 19, 147-152.

[9] Popa, V. and Noiri, T. (2000) On m-Continuous Functions. Annals of the University Dunarea de Jos of Galati, 18, 31-41.

[10] Noiri, T. and Popa, V. (2000) On Upper and Lower $m$-Continuous Multifunctions. Filomat, 14, 73-86.

[11] Banerjee, K.A. and Saha, K.P. (2015) Semi Open Sets in Bispaces. Cubo (Temuco), 17, 99-106. https://doi.org/10.4067/S0719-06462015000100008

[12] Kelly, J.C. (1963) Bitopological Spaces. Proceedings of the London Mathematical Society, 3, 71-89. https://doi.org/10.1112/plms/s3-13.1.71

[13] Min, W.K. (2009) m-Semiopen Sets and $m$-Semicontinuous Functions on Spaces with Minimal Structures. Honam Mathematical Journal, 31, 239-245.

https://doi.org/10.5831/HMJ.2009.31.2.239 